\title{
EDITORIAL
}

\section{Editorial zum Schwerpunktthema: Schnittstellenarbeit für die Schulsystementwicklung}

\section{Editorial to the Focus Topic: Interface Activities for School System Development}

Die Frage nach gelingendem Wissenstransfer und Zusammenarbeit der verschiedenen Institutionen im Bildungsbereich (wie Schule, Wissenschaft und Bildungsverwaltung) ist eine alte Frage, die sich immer wieder an den Herausforderungen des „TheoriePraxis-Problems“ entzündet. Vor allem ist aus Steuerungsperspektive der Ruf danach zu verzeichnen, Strategien und Verfahren zu konzipieren und zu realisieren, die zu einer Verarbeitung und Nutzung von Forschungswissen in der Weise beitragen, dass ein Mehrwert für die schulsystemische Qualitätsentwicklung entsteht (z. B. KMK, 2015). Gleichzeitig ist $\mathrm{zu}$ beobachten, dass sich von einseitigen Steuerungsvorstellungen nach dem Muster „Wissenschaft stellt der Politik/Bildungsverwaltung Angebote bereit - die Steuerungsakteure greifen diese zur Etablierung von Qualitätsentwicklungsmaßnahmen auf" dahingehend verabschiedet wird, dass Wissenstransfer und Wandel im Bildungssystem eben nicht derart linear funktionieren, sondern kooperativer Konstellationen, transformativer Verarbeitungsprozesse und einer reflexiven Wissensverarbeitung bedürfen (Bieber, Egyptien, Klein, Oechslein \& Pikowsky, 2018). Dies rückt somit die Frage in den Blick, welche Akteure in welcher Funktion in diesen Konstellationen involviert oder noch einzubinden sind, welche transformativen Leistungen sie erbringen (können) und wie letztlich die Arbeit an diesen „Schnittstellen" ausgestaltet wird.

Folglich erscheint es erforderlich, nach kommunikativen Verknüpfungen zu suchen, die den Informationsfluss zwischen Schulpraxis, Bildungsverwaltungspraxis (dies meint die verschiedenen Institutionen des Regelsystems wie Ministerien, Landesinstitute, Fortbildungssystem, Schulaufsicht etc.) und Forschung begünstigen. Zu fehlen scheint in diesem Zusammenhang eine deutlich breitere Rezeption des schon vorliegenden internationalen Forschungsstandes - beispielsweise von Arbeiten, die dezidiert die in Transferprozessen eingebundenen Akteurskonstellationen sowie die damit verknüpften Schnittstellen und Interdependenzen analysieren und Implikationen für Modell- und Theoriebildung liefern (z.B. Malin \& Brown, 2020; Ward, 2017; Farley-Ripple, Tielley \& Tise, 2017; Ng-A-Fook, Kane, Butler, Glithero \& Forte, 2015; 
Cooper, 2013). Dass hier noch Bedarf an Aufarbeitung besteht, zeigt exemplarisch bereits die Tatsache, dass die schulsystemischen Institutionen in Deutschland, die die Einzelschulen unterstützen sollen und denen rein programmatisch eine wichtige Rolle in Transferprozessen zugewiesen wird, bislang wenig erforscht werden (z. B. Schulaufsicht und Landesinstitute).

Die Forschungsdesiderate im deutschsprachigen Raum liegen zum einen im Mangel an empirisch fundierten Erkenntnissen und zum anderen auch in einer auf schulsystemische Fragestellungen fokussierten theoretischen Auseinandersetzung begründet, die sich dezidiert mit den „Schnittstellen“ der Institutionen beschäftigt, die entweder für die Erfüllung des staatlichen Erziehungs- und Bildungsauftrages zuständig sind (Schule) oder aber auf diesen Auftrag ausgerichtet entsprechende Unterstützungsleistungen erbringen sollen oder dies - selbst gewählt - leisten wollen (Fortbildung, Schulaufsicht, Ministerien, Landesinstitute, Schulträger, Stiftungen, kommunale Partner usw.). Konkret braucht es Forschungen, die vermehrt die Ausgestaltung der interinstitutionalen Schnittstellen, also die kooperativen Konstellationen zwischen systemisch-strukturell verflochtenen Akteuren (z. B. Schule und Schulaufsicht) oder aber die initiativ-programmatischen Verknüpfungen (z.B. Schule und Stiftungen) in den Blick nehmen und diese Formen der Zusammenarbeit nicht nur deskriptiv erfassen, sondern letztlich auch auf ihre Wirksamkeit für schulische Qualitätsentwicklung hin befragen. Dies impliziert auch ein Verständnis eines zu untersuchenden Institutionengefüges, das mit seinem „Unterstützungsfokus“ auf die Qualität der schulischen Praxis auch selbst der qualitativen Weiterentwicklung bedarf. Dies meint letztlich Schulsystementwicklung - die gemeinsame Anstrengung von für das System funktional zuständigen sowie weiteren sich einbringenden Akteuren, zur schulischen Qualitätsentwicklung (unterstützend) beizutragen und aus diesem Anspruch heraus auch sich selbst zum Gegenstand von Forschung zu machen, um eine gesamtsystemische Entwicklung zu befördern.

Doch ungeachtet der zuvor angeführten Desiderate sollen die vorhandenen Ansätze und empirischen Explorationen, die auch im deutschsprachigen Raum vorliegen, nicht unterschlagen werden. Es ist sicherlich der bildungsbezogenen Governanceforschung (Altrichter, Brüsemeister \& Wissinger, 2007; Heinrich, 2007) zu verdanken, dass sie mit ihrem Fokus auf die vorhandene Vielfalt an Akteuren im Schulsystem deren regulative Verflechtungen untereinander und die jeweiligen Interdependenzen prominent in einen Forschungsfokus gerückt hat. Auch hat die schulbezogene Netzwerkforschung (Berkemeyer \& Bos, 2010) in den letzten gut 20 Jahren Zuwachs erfahren, was allein theoretische Modellierungen, aber auch die Zahl empirischer Arbeiten verdeutlichen. Hier lassen sich vor allem Beiträge ausmachen, die insbesondere die Schule in den Blick nehmen (z. B. die interschulische Vernetzung oder aber - unter Einbezug kommunaler Akteure - die Forschung zum schulischen Übergangsmanagement). Als ein weiterer disziplinärer Zugang zu dem Zusammenspiel von Akteuren kann die PolicyForschung gelten. Diese schaut aus politikwissenschaftlicher Perspektive auf die ver- 
schiedenen Logiken vor allem politischer Bemühungen, Innovationen im System anzustoßen und erfolgreich zu implementieren (z. B. Viennet \& Pont, 2017).

Der Themenschwerpunkt dieses Heftes versucht mit seinen Beiträgen, der Idee von einer die Schulsystementwicklung beeinflussenden Schnittstellenarbeit näherzukommen. Dabei wird von den Herausgeber*innen zweierlei intendiert: Zum einen soll mit den Beiträgen ein Angebot theoretisch-konzeptioneller Verständnisweisen von Schnittstellen und ihrer Ausgestaltung offeriert und zum anderen sollen empirische und praktische Erkenntnisse aus entsprechenden Projektkonstellationen sowie Erfahrungen aus den US-amerikanischen und kanadischen Diskursen dargelegt werden:

Björn Hermstein bietet zunächst eine grundständige Klärung des Begriffs der Schulsystementwicklung als ein „intermediäres Handlungsfeld“ an, womit die potenzielle Vielfalt an Akteuren, Ereignissen und erwartbar differenten Logiken, aber auch die Diffusität dieses Gegenstandsbereich angedeutet werden. Unter Rückgriff auf den Ansatz des Strukturtheoretischen Individualismus konzipiert er weiter das Konstrukt von Schnittstellen und geht unter Einbezug empirischer Erkenntnisse der Frage nach, wie sich Schnittstellen rund um den Akteur Schulträger konstituieren und in der Schulsystementwicklung zur Geltung kommen.

Mit Anja Jungermanns Beitrag werden ein spezifisches Konzept und seine praktische Anwendung in der Zusammenarbeit von Akteurskonstellationen mit dem Ziel einer gemeinsam verstandenen und getragenen Ausrichtung von qualitätsorientierter Entwicklungsarbeit im Schulsystem vorgestellt. Es handelt sich hierbei um die „wirkungsorientierte Steuerung als Kommunikationsinstrument“, deren praktische Anwendung die Autorin zudem mithilfe von Erkenntnissen aus einer Fallstudie illustriert.

William R. Penuel, Erin Marie Furtak und Caitlin C. Farrell geben Einblicke in den amerikanischen Diskurs zu den „Research-Practice Partnerships“. Ausgehend von der Notwendigkeit einer „institutional logic of evolution“ wird das Erfordernis des kollektiven Lernens innerhalb von gemeinsamen Unterfangen, wie etwa der Schulsystementwicklung, postuliert. Diese Argumentation wird mithilfe der Erkenntnisse aus den Forschungsarbeiten der Forscher*innen zu Research-Practice Partnerships plastisch ausgeführt. Hierbei werden auch gebündelt die Potenziale und Herausforderungen solcher Konstellationen bilanziert und im Hinblick auf den deutschsprachigen Raum diskutiert.

Schließlich geben zwei Berichte Einblicke in die konkrete Arbeit an den Schnittstellen der Schulsystementwicklung. Aus seiner Erfahrung in der Mitarbeit in dem Projekt „Ganz In“ berichtet Wendel Hennen aus Nordrhein-Westfalen, wie in diesem Projekt das Ziel verfolgt wurde, Projekterkenntnisse in die Strukturen des Regelsystems 
(in diesem Fall das Fortbildungssystem) zu überführen. Die dafür genutzte spezifische Konstellation der „Transferarbeit“ von Wissenschaft, Landesinstitut und Schulpraktiker ${ }^{\star}$ innen wird erläutert, und die damit gesammelten Erfahrungen werden aus Sicht des Autors kritisch reflektiert. Mit dem Beitrag von Carol Campbell werden umfassende Erfahrungen mit partnerschaftlichen Formen der Zusammenarbeit zur qualitativen Weiterentwicklung des Schulsystems aus Ontario berichtet. Insgesamt geht es um die Erkenntnisse aus zwei Phasen reformerischer Bemühungen um partnerschaftliche Zusammenarbeit - die eine mit dem Fokus auf die Verbesserung der Leistung von Schüler*innen und die darauffolgende mit dem Schwerpunkt auf eine kooperativ ausgerichtete Professionalisierung, die an Gerechtigkeitsfragen orientiert ist. Die Autorin bietet zudem eine kritische Diskussion zu bislang vorliegenden Erfahrungen der Reformbemühungen in Ontario und zieht Schlussfolgerungen angesichts aktueller Herausforderungen, wie etwa der COVID-19-Pandemie.

\section{Veronika Manitius, Götz Bieber \& Nina Bremm}

\section{Literatur und Internetquellen}

Altrichter, H., Brüsemeister, T., \& Wissinger, J. (2007). Educational Governance. Handlungskoordination und Steuerung im Bildungssystem. Wiesbaden: VS. https://doi. org/10.1007/978-3-531-90498-6

Berkemeyer, N., \& Bos, W. (2010). Netzwerke als Gegenstand erziehungswissenschaftlicher Forschung. In C. Stegbauer \& R. Häußling (Hrsg.), Handbuch Netzwerkforschung (S. 755-770). Wiesbaden: Springer. https://doi.org/10.1007/978-3-531-92575-2_66

Bieber, G., Egyptien, E., Klein, G., Oechslein, K., \& Pikowsky, B. (2018). Positionspapier der Landesinstitute und Qualitätseinrichtungen der Länder zum Transfer von Forschungswissen. Zugriff am 30.12.2020. Verfügbar unter: https://www.qua-lis.nrw.de/cms/up load/aktuelles/Positionspapier_Transfer_31.10.18.pdf.

Cooper, A. (2013). Research Mediation in Education: A Typology of Research Brokering Organizations That Exist Across Canada. Alberta Journal of Educational Research, 59 (2), 181-207.

Farley-Ripple, E., Tielley, K., \& Tise, J. (2017). Brokerage and the Research-Practice Gap: A Theoretical and Empirical Examination. Paper Presented at the 2017 Annual Meeting of the American Educational Research Association.

Heinrich, M. (2007). Governance in der Schulentwicklung. Von der Autonomie zur evaluationsbasierten Steuerung. Wiesbaden: VS.

KMK (Sekretariat der Ständigen Konferenz der Kultusminister der Länder in der Bundesrepublik Deutschland). (2015). Gesamtstrategie der Kultusministerkonferenz zum Bildungsmonitoring. Beschluss der 350. Kultusministerkonferenz vom 11.06.2015. Berlin: KMK. Zugriff am 20.12.2020. Verfügbar unter: https://www.kmk.org/fileadmin/ Dateien/pdf/Themen/Schule/Qualitaetssicherung_Schulen/2015_06_11-Gesamtstrate gie-Bildungsmonitoring.pdf.

Malin, J., \& Brown, C. (2020). The Role of Knowledge Brokers in Education. Connecting the Dots Between Research and Practice. London \& New York, NY: Routledge. https://doi. org/10.4324/9780429462436

Ng-A-Fook, N., Kane, R. G., Butler, J. K., Glithero, L., \& Forte, R. (2015). Brokering Knowledge Mobilization Networks: Policy Reforms, Partnerships, and Teacher 
Education. Education Policy Analysis Archives, 23 (122). https://doi.org/10.14507/epaa. v23.2090

Viennet, R., \& Pont, B. (2017). Education Policy Implementation: A Literature Review and Proposed Framework. OECD Education Working Papers, No. 162. Zugriff am 30.12.2020. Verfügbar unter: http://www.oecd.org/officialdocuments/publicdisplaydoc umentpdf $/$ cote $=$ EDU $/ W K P(2017) 11 \&$ docLanguage $=$ En .

Ward, V. (2017). Why, Whose, What and How? A Framework for Knowledge Mobilisers. Evidence \& Policy, 13 (3), 477-497. https://doi.org/10.1332/174426416X14634763 278725

https://doi.org/10.31244/dds.2021.01.02 